\title{
Effect of Combination Therapy with Sodium Ozagrel and Panax Ginseng on Transient Cerebral Ischemia Model in Rats
}

\author{
Sang In Park, ${ }^{1}$ Dong-Kyu Jang, ${ }^{1}$ Young-Min Han,, ${ }^{1}$ Yun-Young Sunwoo, ${ }^{1}$ \\ Moon-Seo Park, ${ }^{1}$ Yong-An Chung, ${ }^{1}$ Lee-So Maeng, ${ }^{1}$ Ruth Im, ${ }^{1}$ Min-Wook Kim, ${ }^{1}$ \\ Sin-Soo Jeun, ${ }^{2}$ and Kyung-Sool Jang ${ }^{1}$ \\ ${ }^{1}$ Institute of Catholic Integrative Medicine (ICIM), Incheon St. Mary's Hospital, The Catholic University of Korea, \\ Incheon 403-720, Republic of Korea \\ ${ }^{2}$ Department of Neruosurgery, College of Medicine, The Catholic University of Korea, Seoul 137-701, Republic of Korea
}

Correspondence should be addressed to Kyung-Sool Jang, jks611@catholic.ac.kr

Received 8 November 2010; Accepted 8 December 2010

Academic Editor: Thomas Van Groen

Copyright $\odot 2010$ Sang In Park et al. This is an open access article distributed under the Creative Commons Attribution License, which permits unrestricted use, distribution, and reproduction in any medium, provided the original work is properly cited.

Sodium ozagrel (SO) prevents platelet aggregation and vasoconstriction in the cerebral ischemia. It plays an important role in the prevention of brain damage induced by cerebral ischemia/reperfusion. Recently, many animal studies have suggested that the Panax ginseng (PG) has neuroprotective effects in the ischemic brain. In this study, we assessed the neuroprotective effects that come from a combination therapy of SO and PG in rat models with middle cerebral artery occlusion (MCAO). Animals with MCAO were assigned randomly to one of the following four groups: (1) control (Con) group, (2) SO group (3 mg/kg, intravenously), (3) PG group (200 mg/kg, oral feeding), and (4) SO + PG group. The rats were subjected to a neurobehavior test including adhesive removal test and rotarod test at $1,3,7,10$, and 15 days after MCAO. The cerebral ischemic volume was quantified by Metamorph imaging software after 2-3-5-triphenyltetrazolium (TTC) staining. The neuronal cell survival and astrocytes expansion were assessed by immunohistofluorescence staining. In the adhesive removal test, the rats of PG or SO + PG group showed significantly better performance than those of the control group (Con: $88.1 \pm 24.8, \mathrm{PG}: 43.6 \pm 11, \mathrm{SO}+\mathrm{PG}$ : $11.8 \pm 7, P<.05)$. Notably, the combination therapy group ( $\mathrm{SO}+\mathrm{PG}$ ) showed better performance than the SO group alone (SO: $56 \pm 12$, SO + PG: $11.8 \pm 7, P<.05)$. In TTC staining for infarct volume, cerebral ischemic areas were also significantly reduced in the PG group and SO + PG group (Con: $219 \pm 32, \mathrm{PG}: 117 \pm 8, \mathrm{SO}+\mathrm{PG}: 99 \pm 11, P<.05)$. Immunohistofluorescence staining results showed that the group which received SO + PG group therapy had neuron cells in the normal range. They also had a low number of astrocytes and apoptotic cells compared with the control or SO group in the peri-infarction area. During astrocytes staining, compared to the SO + PG group, the PG group showed only minor differences in the number of NeuN-positive cells and quantitative analysis of infarct volume. In conclusion, these studies showed that in MCAO rat models, the combination therapy with SO and PG may provide better neuroprotective effects such as higher neuronal cell survival and inhibition of astrocytes expansion than monotherapy with SO alone.

\section{Introduction}

Acute ischemic stroke is one of the leading causes of adult disability and death in the world, with an incidence affecting up to $0.2 \%$ of the population every year [1]. Neuronal cell injury, including apoptosis, is the major event in the acute and subacute phase of cerebral ischemia, where the formation of a glial scar resulting from reactive gliosis (mainly consisting of proliferated astrocytes) is detected in the late phase $[2,3]$. Reactive gliosis plays the role of a biochemical and physical barrier for regeneration of the axon [2]. Although the exact mechanism of neurological destruction of the brain caused by cerebral ischemia has not been elucidated yet, studies suggest that oxidative stress, inflammatory cytokines, and excitotoxicity may play a role $[4,5]$.

Many researchers suggested that these mechanisms destruct the neuronal cells and induce the vasoconstriction of 
cerebral artery. Platelet aggregation was also reported as one of the aggravating factors of cerebral ischemia. The role of thromboxane A2 (TXA2), a strong vasoconstrictor and platelet aggregator, was well established by several studies as an example, [6-9]. Sodium ozagrel (SO), a selective TXA2 synthetase inhibitor, showed a suppressive effect on vasospasm and platelet aggregation in animal and human experiments $[8,9]$.

Traditionally, the root of Panax ginseng (PG) has been widely used as a herbal medicine to treat diverse diseases including some neurological disorders in Korea. Yoshikawa and his colleague reported that PG improves neurological dysfunction and prevents histological injury such as proliferation of astrocyte and apoptosis of neuronal cells in the ischemic brain [10]. Also, some researchers showed decrease in the infarct volume after administration of PG extract in the cerebral ischemic rat model $[11,12]$. They suggested that the roots of PG have a neuroprotective effect in the ischemic brain, caused by inflammation inhibition and microglial activation. These anti-inflammation, antioxidation, antithrombotic, and antiapoptotic effects on neuronal cells prompt us to test effects of PG extract on the recovery from cerebral ischemia. These days, it has become a very common practice to take herbal medicine. In the United States, nearly 1 in 6 adults concomitantly take at least one herbal remedy with their prescription drugs [13]. Recently, many western studies have started trying combination therapy with herbal medicine especially for cerebral ischemia.

In this study, we assessed whether the combination therapy of SO and PG shows synergistic or additive effect in the recovery of cerebral ischemia by performing a neurobehavioral test and histological observation on infarct areas.

\section{Materials and Methods}

2.1. Preparation of SO and Administration to Rats. SO (Kissei Pharmaceutical Co. Ltd., Matsumoto, Japan), $4 \mathrm{mg} / \mathrm{ml}$, was prepared by dissolving in $0.9 \%$ saline. SO was injected intravenously to the tail vein within $30 \mathrm{~min}$ after the reperfusion in the middle cerebral artery occlusion (MCAO) rat models. Rats were treated with SO solution ( $3 \mathrm{mg} / \mathrm{kg}$ ) everyday for a week [7].

\subsection{Preparation of $P G$ Extracts and Administration to Rats.} Dried roots of $P G$ were obtained from the National Institute of Crop Science and identified by Dr. Kim, Ginseng \& Medicinal Crops Division, National Institute of Crop Science, Rural Development Administration (Suwon, Republic of Korea). Powdered extracts of PG were made as described by Kim et al. [14]. Dried roots of PG $(1.0 \mathrm{~kg})$ were extracted with $70 \%$ ethanol under sonication. The $70 \%$ ethanol filtrate was evaporated in vacuo to give ethanol extracts $(238 \mathrm{~g})$, which were then stored at $-20^{\circ} \mathrm{C}$ until use. The powdered extracts of PG were dissolved in saline to obtain a solution of $200 \mathrm{mg} / \mathrm{mL}$. Animals were fed the same doses of PG through the oral zonde needle within $30 \mathrm{~min}$ after the reperfusion in the rat models $(200 \mathrm{mg} / \mathrm{kg})$ [14]. Rats were treated everyday for a week with $\mathrm{PG}$ extracts.

2.3. Ischemic Animal Model. All animal procedures were carried out in accordance with the animal experimental guideline established by the Institutional Animal Care and Use Committee of Catholic University Medical School. Transient MCAO was induced as described by Longa et al., [15] with a minor modifications. Male Sprague-Dawley rats (270-305g) were briefly anesthetized with 5\% isoflurane in oxygen/nitrogen (N2O/O2 [30/70]). During a surgical procedure, a mixture of $2 \%$ isoflurane and N2O/O2 (30/70) was used to maintain anesthesia. Rectal temperature was maintained at $\left(37 \pm 5^{\circ} \mathrm{C}\right)$ using a rectal thermometer and a heating pad (Harvard Apparatus Inc., Holliston, Massachusetts, USA). The right common carotid artery (CCA), external carotid artery (ECA), and internal carotid artery (ICA) were exposed through a ventral midline incision. A 3-0 monofilament nylon suture with a rounded tip was introduced into the CCA lumen and gently advanced into the ICA until it blocked the bifurcating origin of the MCA.

Cortical blood flow was measured continuously with a laser Doppler (Transonic system Inc., Ithaca, NY, USA) in anesthetized animals. A photodetector probe $(0.45 \mathrm{~mm}$ in diameter) was stereotaxically placed through a skull burr hole $(1 \mathrm{~mm}$ diameter $)$ in the frontoparietal cortex $(1.3 \mathrm{~mm}$ posterior, $2.8 \mathrm{~mm}$ lateral to the bregma, and $1.0 \mathrm{~mm}$ above the dura). Ninety minutes after the occlusion, animals were reanesthetized and reperfused by withdrawing the suture until the tip cleared the lumen of CCA. Regional cerebral blood flow ( $\mathrm{rCBF}$ ) was expressed as a percentage of preischemic baseline values. MCAO animals were only included if occlusion caused a decrease in $\mathrm{rCBF}$ to $30 \%$ of the original blood flow.

2.4. Experimental Groups. Animals were assigned randomly to one of the following four groups: (1) the first group of rats were treated with saline as the control group $(n=5$, Con group), (2) the second group was injected with SO $(n=5$, SO group), (3) the third group was fed with PG $(n=5, \mathrm{PG}$ group), and (4) the fourth group was treated with $\mathrm{SO}$ plus $\mathrm{PG}(n=5, \mathrm{SO}+\mathrm{PG}$ group $)$.

2.5. Behavior Evaluation. Behavior parameters were evaluated with adhesive removal and rotarod test at $1,3,7,10$, and 15 days after MCAO.

2.6. Adhesive Removal Test. Square dots of adhesive-backed paper $(12 \phi)$ were used as bilateral tactile stimuli occupying the distal-radial region on the wrist of each forelimb. Animals were given three trials with a cut-off time of 180 seconds. The data are presented at the mean time to remove the left dot.

2.7. Rota-Rod test. In the Rota-rod motor test, Animals were pretrained three times for a week before MCAO. The Rotarod cylinder was accelerated from 4 to $40 \mathrm{rpm}$ within 5 minutes, and the cutoff time was 300 seconds. 


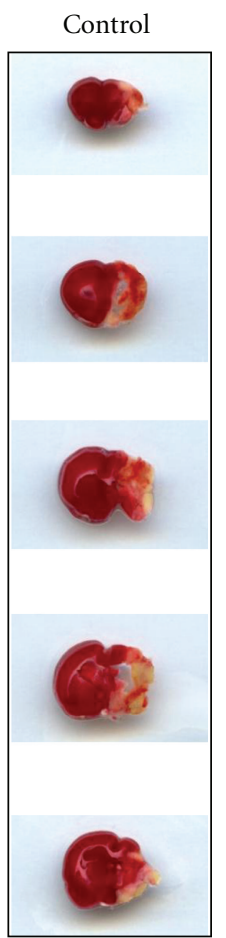

(a)

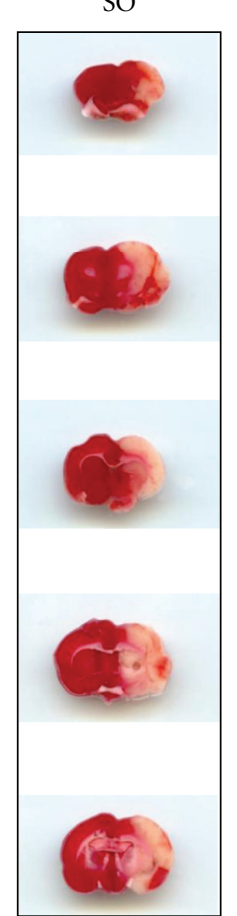

(b)

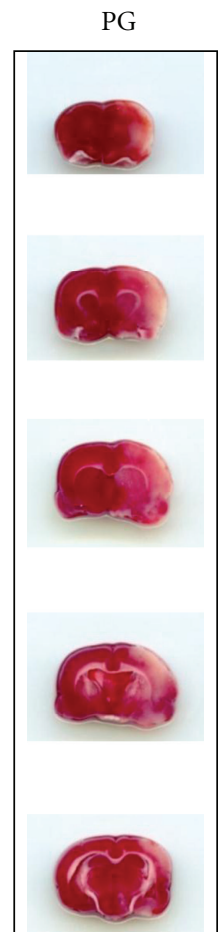

(c)

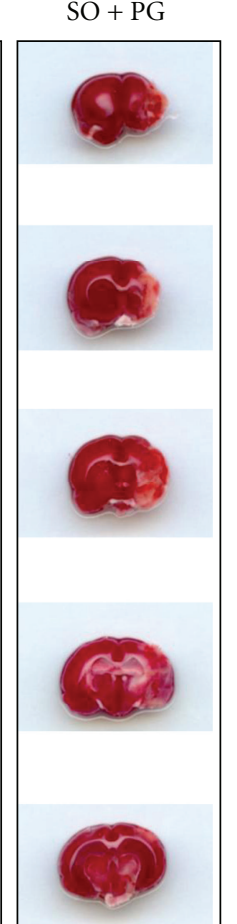

(d)

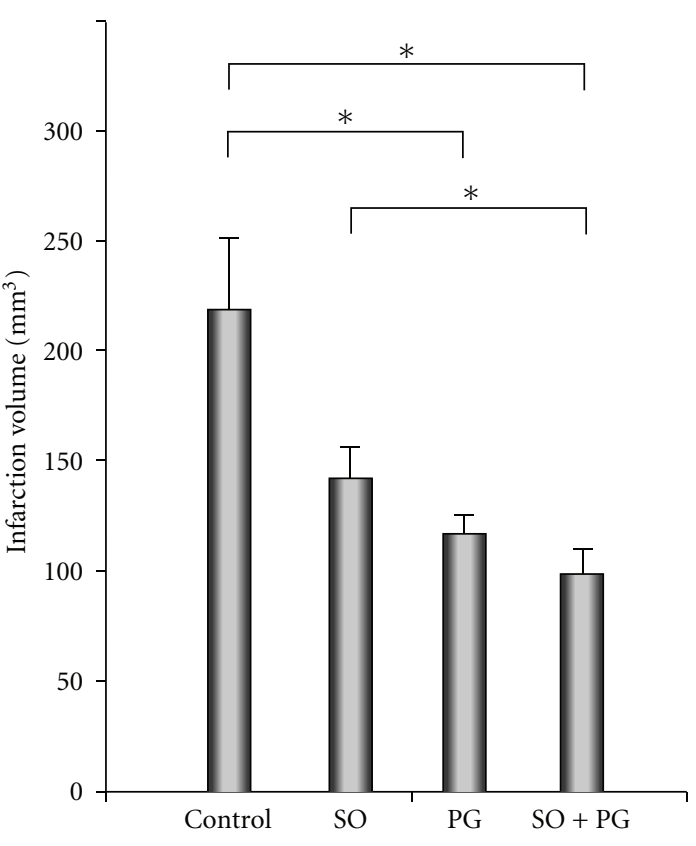

(e)

FIgURE 1: These figures show the cerebral ischemic area at 15 days after MCAO rats. ((a) control group: Con, (b) Sodium Ozagrel group: SO, (c) Panax ginseng group: PG, (d) Sodium Ozagrel plus Panax ginseng group: SO + PG). The ischemic areas were identified by $2 \%$ 2-3-5-triphenylterazolium staining; nonstained areas are ischemic areas. (e) Calculated infarct volumes based on 6 sequential brain slices. Treatment with PG or SO + PG group showed significant reduction in infarct volume, and SO + PG group showed much reduction of infarct volume compared to other groups. ${ }^{*} P<.05$.

2.8. Determination of Infarct Volume. At 15 days after MCAO, all rats ( $n=5$, for each group) were deeply anesthetized with $15 \%$ urethane and sacrificed by decapitation. Then the brain was immediately removed and sectioned into 2 equally spaced $(2 \mathrm{~mm})$ coronal blocks using a rodent brain matrix. These sections were stained by $0.1 \mathrm{M}$ PBS containing 2\% 23-5-triphenylterazolium (TTC; Sigma, St. Louis, MO, USA) for 15 minutes at $37^{\circ} \mathrm{C}$. Using Meta-Morph imaging program (Molecular Devices Inc, Downingtown, PA, USA), the infarct area was determined by using the method of subtracting the area of the noninfarct ipsilateral hemisphere from that of the contralateral tissue to reduce errors that might occur due to cerebral edema.

2.9. Immunohistochemical Staining. The tissue was cut coronally at a thickness of $4 \mu \mathrm{m}$ between $+0.1-0.86 \mathrm{~mm}$ of the boundary area of cerebral infarct in the precentral gyrus, located $24-\mathrm{mm}$ posterior to the frontal pole from each rat for a total of three blocks. The sections were warmed for 20 minutes and washed with $0.01 \mathrm{M}$ PBS for 10 minutes. Sections were blocked in normal goat serum for 1 hour at room temperature. The sections were incubated with mouse antibody to NeuN (Neuronal Nuclei, Millipore, Inc., diluted at 1:100) and GFAP (Glial fibrillary acidic protein, Millipore, Inc., diluted at $1: 200$ ) overnight at $4^{\circ} \mathrm{C}$. Subsequently, sections were incubated for $1 \mathrm{hr}$ at room temperature with Alexa 488-conjugated goat antimouse IgG (Vector Laboratories, $1: 200$ ). After washing, the sections were counterstained and stored at $-20^{\circ} \mathrm{C}$ and then observed under both a fluorescence microscope equipped with a spot digital camera and a confocal scanning laser microscope (LSM 510, Zeiss, Germany). Apoptosis was detected by the terminal deoxynucleotidyl transferasemediated d-UTP-biotin nick end (TUNEL) assay by means of the in situ cell death detection kit (Rochcce, Indianapolis, IN, USA) developed using the Cy2-conjugated streptavidin (Jackson Laboratories, West Grove, PA). The slides were observed by confocal scanning laser microscope. Using MetaMorph imaging program (Molecular Devices Inc, Downingtown, PA, USA), ischemic penumbra was determined by optical density of GFAP and TUNEL and NeuN positive cells.

2.10. Statistical Analysis. The behavior tests, cerebral ischemic volume, tissue density of astrocyte, cell count of NeuN-positive cells, and cell count of apoptotic cells were subjected to the Kruskall-Wallis test and Mann-Whitney $U$ test for each group of rats. Data are presented as mean values \pm standard error. Value of $P<.05$ were considered statistically significant. 


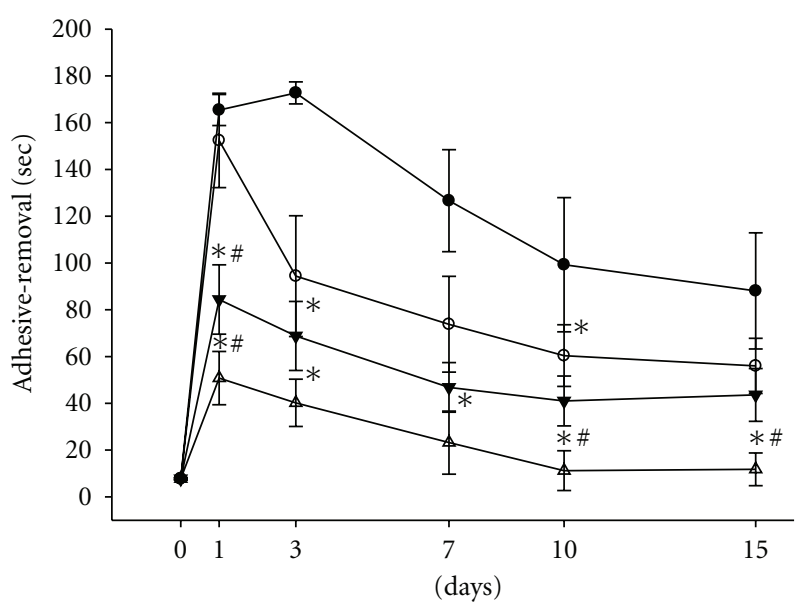

(a)

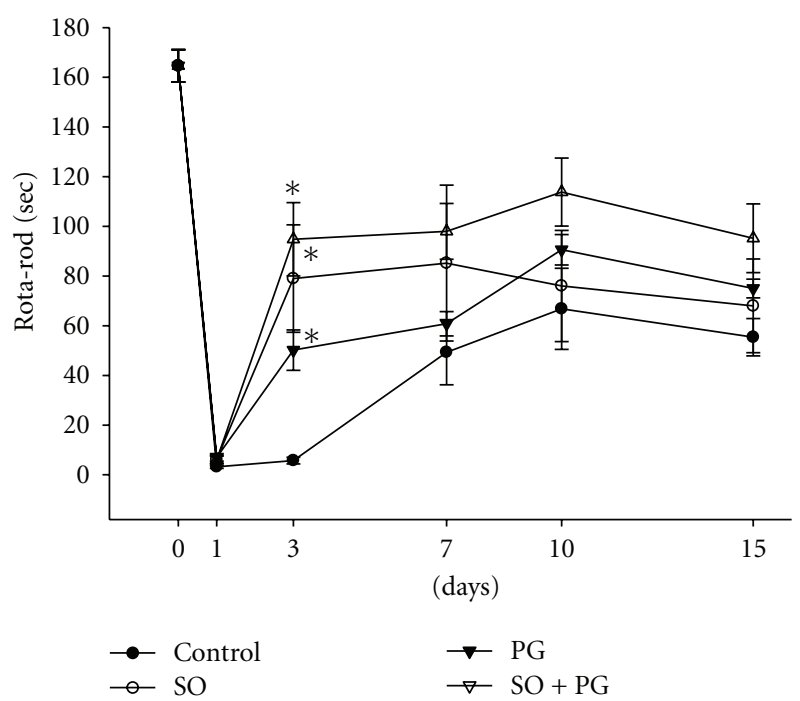

(b)

Figure 2: Behavior test at 15 days after stroke. Adhesive removal test (a) and rotarod test (b) at 1, 3, 7, 10, and 15 days after middle cerebral artery occlusion (MCAO). The group which received combined therapy of SO and PG scored best behavioral performance in both the adhesive removal test and rota-rod test. $* P<.05$.

\section{Results}

3.1. Induction of Transient Cerebral Ischemia Using Laser Doppler. Cortical blood flow measured using a laser-doppler flowmetry (LDF) decreased after MCAO by at least $70 \%$ in all animals. After insertion of the intraluminal filament, the mean $\mathrm{rCBF}$ (regional cerebral blood flow) decreased to less than $24.5 \%$ of the preocclusion value (before MCAO: $63.5 \pm$ $15.4 \mathrm{ml} / 100 \mathrm{~g} / \mathrm{min}, \mathrm{MCAO}: 15.6 \pm 5.16 \mathrm{ml} / 100 \mathrm{~g} / \mathrm{min})$. Immediately after reperfusion, the LDF reading was returned to slightly less than baseline.

3.2. Infarct Volume. The neuroprotective effects of the $\mathrm{SO}+$ PG therapy were evaluated by measuring infarct volumes 15 days after MCAO. The brains of rats were stained with
TTC to obtain infarct volume and calculated by measuring the area of infarction area. Drug-induced hemorrhagic complication was not seen in the rats. Figures $1(\mathrm{a})-1(\mathrm{~d})$ shows typical photographs of TTC-stained sections of treatment and control groups. As seen in Figure 1(e), the infarct volumes of all treatment groups were grossly decreased compared with a control group. But, there were significant differences between control groups and PG group or $\mathrm{SO}+$ $\mathrm{PG}$ group, and also between $\mathrm{SO}$ and $\mathrm{SO}+\mathrm{PG}$ group (Con: $219 \pm 32$, SO: $142 \pm 14$, PG: $117 \pm 8$, SO + PG: $99 \pm 11 \mathrm{~mm}^{3}$, $P<.05)$.

3.3. Behavior Analysis. We evaluated the effects of treatments on the recovery of behavioral functions using adhesive removal and rota-rod test (Figure 2). Prior to MCAO, neurological scores were similar among all groups. As shown in Figure 2(a), PG group and combination therapy with SO and PG group showed significantly improved performance than control group on adhesive removal test (Con: $88.1 \pm$ 24.8, PG: $43.6 \pm 11$, SO + PG: $11.8 \pm 7$ seconds, $P<$ $.05)$. Between SO and PG groups, there was no significant difference in the score of adhesive removal test (SO: $56 \pm$ 12 seconds, PG: $43.6 \pm 11, P<.05)$. Additionally, the group treated with combination of $\mathrm{SO}$ and $\mathrm{PG}$ showed significantly better score than the group treated with $\mathrm{SO}$ alone (SO: $56 \pm 12$, SO + PG: $11.8 \pm 7$ seconds, $P<$ $.05)$. To evaluate coordination, balance, and motor function, rats were subjected to rota-rod test. Only the group treated with combination of SO and PG significantly showed better performance than control group on the results at 3 days (Con: $6 \pm 1$, SO + PG: $95 \pm 15$ seconds, $P<.05$ ). These results show that the group with combined therapy of SO and PG had the best recovery from MCAO.

3.4. Activating Astrocyte, Neuronal Death, and Apoptotic Cell Death. We observed the number of astrocytes, neurons, and apoptotic cells at 15 days after MCAO in the peri-infarct regions. On analysis of GFAP positive cells, all drug-treated groups showed significant decrease in the density of GFAP positive cells compared to the control group (Con: $1455 \pm$ 150, SO: $1085 \pm 121$, PG: $955 \pm 92$, SO + PG: $778 \pm 87$ optical density, $P<.05$ ) (Figure 3 ). There was no difference between SO- and PG-treated groups (SO: $1085 \pm 121, \mathrm{PG}$ : $955 \pm 92$ optical density, $P<.05)$. However, the group treated with combination of SO and PG showed significantly lower astrocyte density compared to the group which was treated with SO or PG alone (SO: $1085 \pm$ 121, PG: $955 \pm$ 92, SO + PG: $778 \pm 87$ optical density, $P<.05)$. This result indicates that $\mathrm{SO}$ and $\mathrm{PG}$ may protect against the delayed infarct expansion through secondary oxidative injury caused by activated astrocytes.

Apoptotic cells were recognized by identification of DNA fragmentation using the terminal deoxynucleotidyl transferase mediated UTP nick end labeling (TUNEL) method. All treated groups had reduced TUNEL-positive cells as compared with control group. Also, the groups treated with combination of SO and PG showed significantly decreased number of TUNEL-positive cells compared with control 


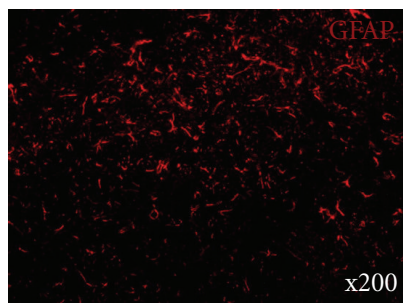

(a)

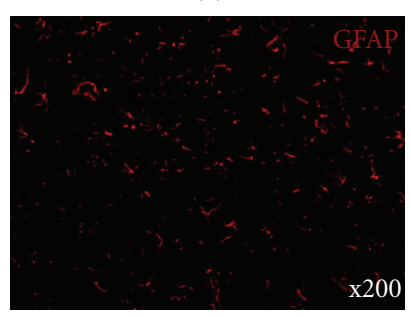

(c)

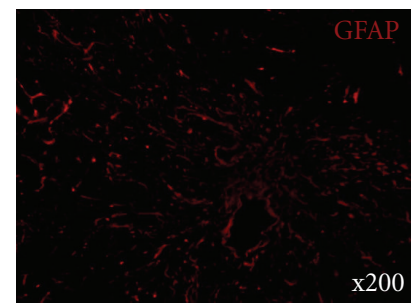

(b)

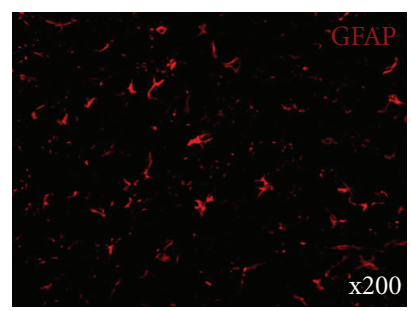

(d)

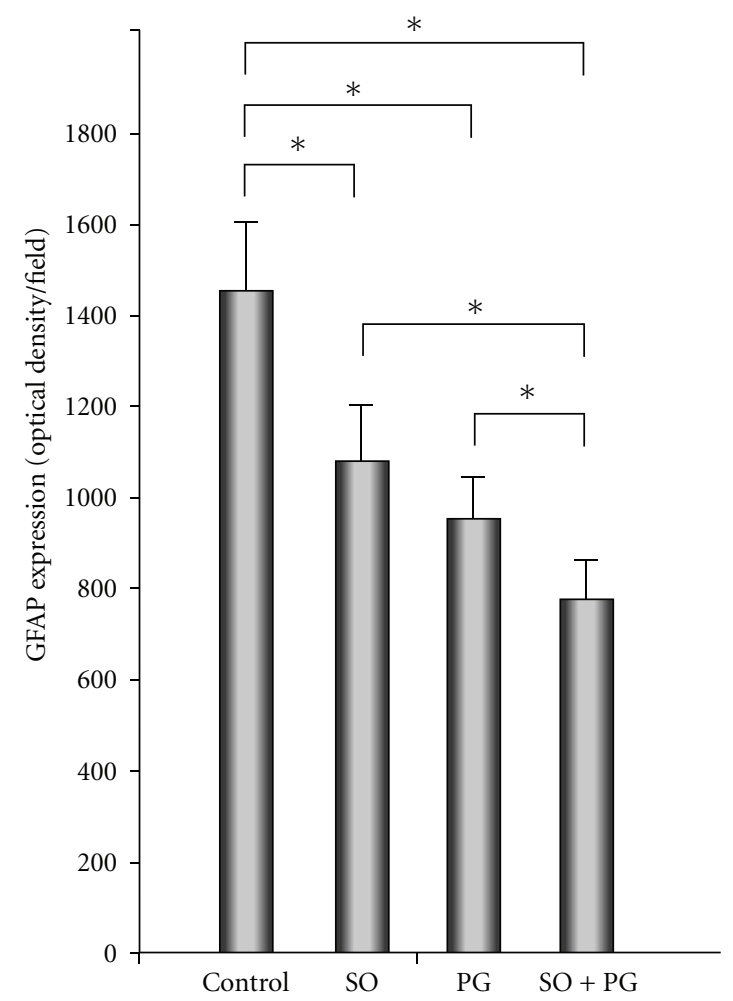

(e)

FIGURE 3: Quantitative analysis of immunoreactivities of GFAP at 15 days after MCAO. Histological analysis of Con (a), SO (b), PG (c), and $\mathrm{SO}+\mathrm{PG}(\mathrm{d})$ was shown into GFAP staining at 15 days after MCAO. Quantitative immunoreactivities for all four groups are shown as bar graphs on the right side of each panel (e). The expression of GFAP in SO + PG group was significantly decreased compared with other groups. ${ }^{*} P<.05$.

group or SO alone (Con: $243 \pm 36, \mathrm{PG}: 135 \pm 10, \mathrm{SO}+$ PG: $114 \pm 8$ cells $\left./ \mathrm{mm}^{2}, P<.05\right)$. However, treatment with SO alone did not show significant decrease in the TUNEL positive cells compared to control group (Con: $243 \pm 36$, SO: $196 \pm 29$, PG: $135 \pm 10$ cells $/ \mathrm{mm}^{2}, P<.05$ ) (Figure 4). In an inverse study of TUNEL stain, all drug-treated groups showed higher number of NeuN-positive cells compared to control group (Con: $36 \pm 9$, SO: $70 \pm 7$, PG: $101 \pm$ 8, SO + PG: $132 \pm 23$ cells $/ \mathrm{mm}^{2}, P<.05$ ) (Figure 5). The group treated with combination of SO and PG showed significantly higher number of NeuN positive cells compared to the SO treated group (SO: $70 \pm 7$, SO + PG: $132 \pm$ 23 cells $\left./ \mathrm{mm}^{2}, P<.05\right)$. These results suggest that $\mathrm{SO}+$ PG group effectively prevents expansion of astrocyte and apoptotic neuronal death in MCAO model.

\section{Discussion}

This study showed for the first time that the combination therapy is superior to monotherapy of SO or PG in poststroke therapy, as evidence by neurobehavioral tests and histological examination. Recently, PG has been applied as treatment for many neurologic disorders [16]. Especially, the effects of free radical scavenger and antiinflammatory functions have been reported to play a role of neuroprotection [12,17, 18]. Ye et al., [19] reported that the ginsengs have neuroprotection effects on animals with cerebral infraction which have insulted by glucoseoxygen deficiency. Kim et al., [14] reported similar results in global ischemic rat models. Recently, Yoshikawa et al., [10] have reported that ginseng have reduced the neurodegeneration around the cerebral infraction areas. In this present study, all drug-treatment groups showed improvement of behavioral disability than control group using the adhesive removal test. Especially, SO + PG group showed significant improvement of behavioral disability in both the rotarod test and adhesive removal test. These behavioral assessment results of $\mathrm{SO}+\mathrm{PG}$ group were outstanding compared to the control group and monotherapy with SO. They suggest that PG attributes to the improvement of neurobehavioral disability in the MCAO rats. These results are similar with what prior researchers have studied [10]. Yoshikawa et al. [10] showed that ginsenoside Rb1, which is a major ingredient of PG, protects the neurological dysfunctions and improve the neurologic score in cerebral ischemic animals.

According to the reports, the ischemic area markedly reduced after treatment of PG $[11,12]$. Son et al., [12] showed decrease in infarction volume after administration of PG extract in MCAO rat models. They suggest that the roots 


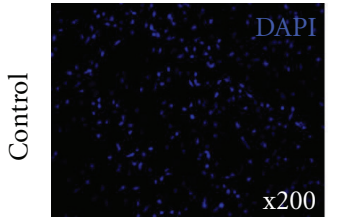

(a)

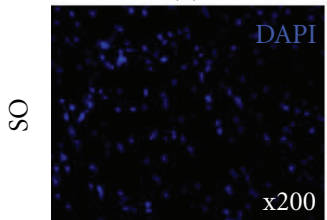

(d)

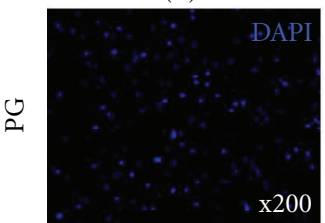

(g)

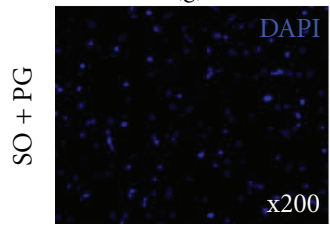

(j)

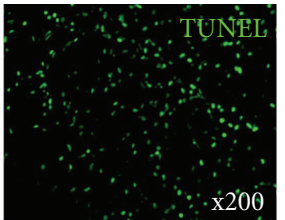

(b)

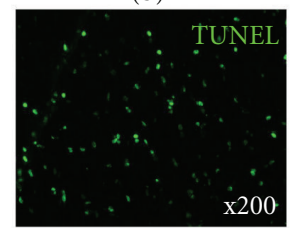

(e)

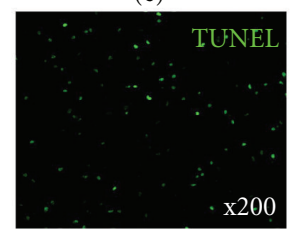

(h)

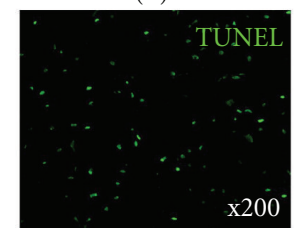

(k)

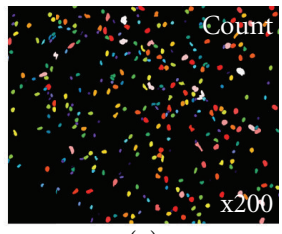

(c)

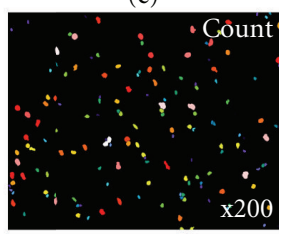

(f)

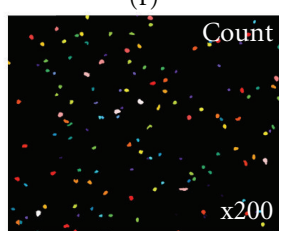

(i)

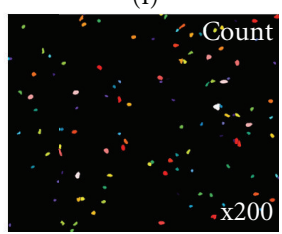

(1)

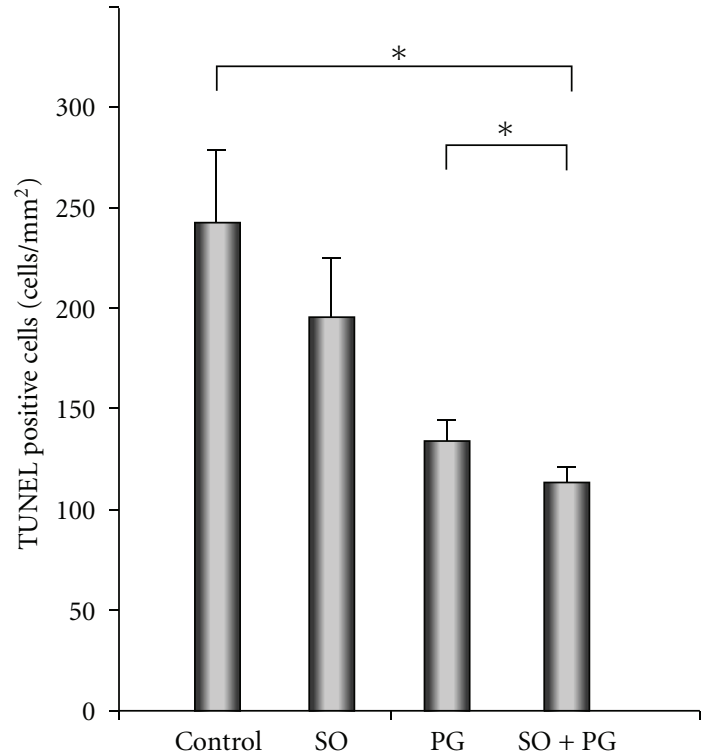

$(\mathrm{m})$

FIGURE 4: Quantitative analysis of the TUNEL staining for identifying apoptotic cells. Apoptotic cells were identified by immunofluorescence FITC (green; (b), (e), (h), (k)) and DAPI (blue; (a), (d), (g), (j)). TUNEL positive cells were counted using Meta-Morph program (c), (f), (i), (l). (m) shows the quantitative data for TUNEL positive cells. The SO + PG group was significantly decreased compared to Con and PG. $* P<.05$.

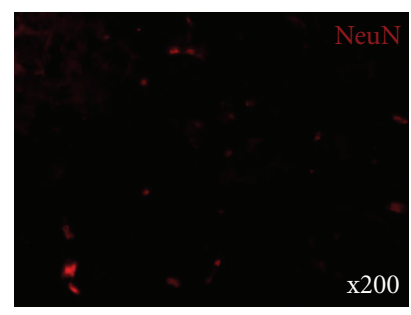

(a)

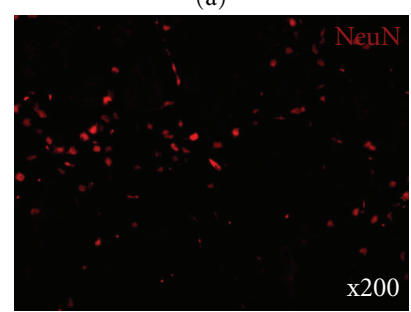

(c)

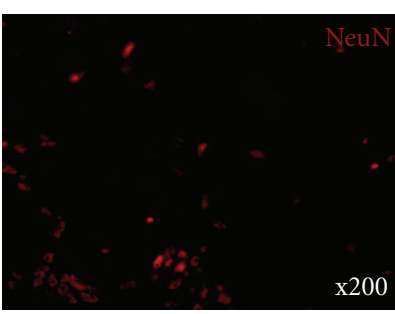

(b)

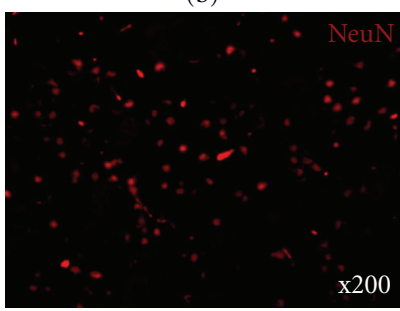

(d)

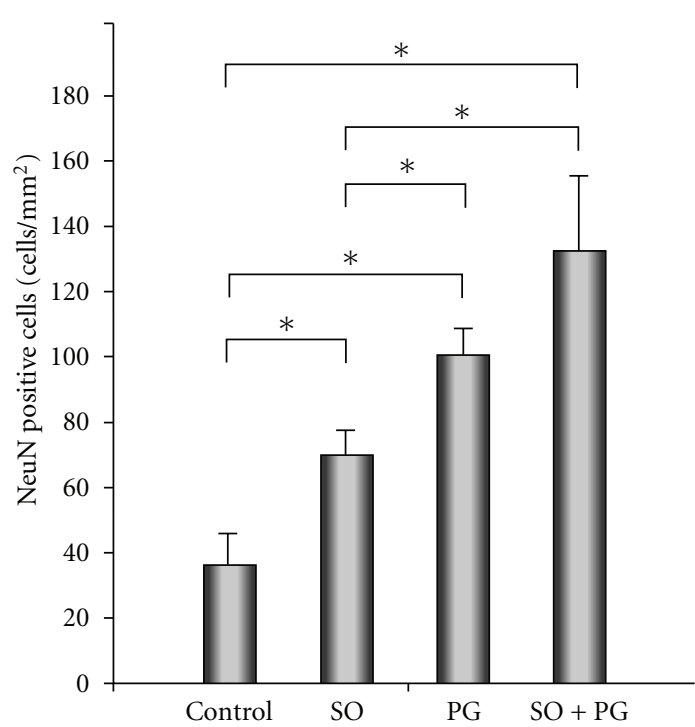

(e)

Figure 5: Quantitative analysis of NeuN-positive cell. These figures show the NeuN positive cells on peri-infarct area in Con group (a), SO group (b), PG group (c), and SO plus PG group (d) at 15 days after MCAO. The number of NeuN positive cells in this area showed significant difference between control group and all treated groups. The number of these cells decreased very much in the control group. There were many NeuN-positive cells in SO + PG group compared to other groups. However, there was no difference between PG and SO + PG group. $* P<.05$. 
of PG have a neuroprotective effect in the ischemic brain. Due to the inhibition of inflammation and of microglial activation, it can be used as a potential therapeutic agent against inflammation in ischemic stroke [12]. In the present study, SO + PG group showed a significant reduction in cerebral infarction area than control or SO group after treatment. However, there was no difference between control and $\mathrm{SO}$ alone, as there were no difference between combination group and PG group. This reveals that the cerebral ischemic reduction effect of PG is superior than SO in cerebral ischemic rats.

These results suggest that PG plays a role as a neuroprotector in the combination treatment group. Immunohistochemical staining data demonstrated that all treated drugs suppressed an increase of GFAP-positive cells in the peripheral area of ischemic infarction, and combinationtreated group was predominant over others. This suggested that combination with SO and PG may protect against the infarct expansion through secondary injury caused by activated astrocytes. Apoptosis is a destructive mechanism in association with neuronal cell death after an ischemic stroke. NeuN antigen had widely been used as a marker of neuronal cell loss [20]. This study showed that the groups treated with combination of SO and PG showed significant decreased number of TUNEL positive cells compared with control group or SO alone group. However, treatment with SO alone did not significantly decrease the TUNEL positive cells compared to control. In the staining of NeuN-positive cells, all drug-treated groups showed higher number of NeuN positive cells compared to control group. The group treated with combination SO and PG showed significantly higher number of NeuN positive cells compared to the SO treated group. These results suggest that combination with PG more effectively prevents expansion of astrocyte and apoptotic neuronal death than SO alone in MCAO model.

Immunohistochemical staining data showed the decreased staining of astrocyte, TUNNEL-positive cell, and increased staining of NeuN-positive cells on the periinfarction area in all the treated groups. However, these results were predominant in the SO + PG group. These findings suggest that the combination group has an antiapoptotic effect in cerebral ischemia in rats and inhibition effect of a glial cell proliferation that results from reactive gliosis.

Platelet activation and subsequent aggregation play one of the key roles in the pathogenic mechanisms of ischemic brain damage [21]. Platelet activation is aggravated after an ischemic stroke $[22,23]$, leading to secondary thrombus formation and further expansion of the cerebral infarction [24]. Many researchers suggested that SO prevents platelet aggregation, vascular constriction, and brain edema in acute cerebral ischemia [6-9]. In the present study, we presumed that the SO may attribute to the inhibition of postischemic hypoperfusion and induce the cerebral edema after the cerebral ischemia/reperfusion via the inhibition of the TXA2 synthesis. But, all of our studies showed that the neuroprotective effects of PG were superior than SO in MCAO rats.

\section{Conclusion}

We have shown that the combination therapy with $\mathrm{SO}$ and PG may provide more of the neuroprotective effects than monotherapy with SO or PG alone in MCAO stroke rat models such as reduction of infarct volume and prevention of gliosis and neuronal cell death. Therefore, the author suggests that the cerebral blood flow increased to the peripheral area of ischemic zone by $\mathrm{SO}$, and several neuroprotective effects of PG make the additive effect in the cerebral ischemic rats. These results suggested that if these two types of medicines are simultaneously used in treating patients with acute cerebral infarction, it could significantly improve the outcome of patients.

\section{Conflict of interest}

The authors report no conflicts of interest.

\section{Acknowledgments}

The research was supported by the Converging Research Center Program through the Ministry of Education, Science, and Technology (2010K001055), and Clinical Research Laboratory of Incheon St. Mary's Hospital, The Catholic University of Korea.

\section{References}

[1] C. J. M. Klijn and G. J. Hankey, "Management of acute ischaemic stroke: new guidelines from the American Stroke Association and European Stroke Initiative," Lancet Neurology, vol. 2, no. 11, pp. 698-701, 2003.

[2] J. W. Fawcett and R. A. Asher, "The glial scar and central nervous system repair," Brain Research Bulletin, vol. 49, no. 6, pp. 377-391, 1999.

[3] J. Silver and J. H. Miller, "Regeneration beyond the glial scar," Nature Reviews Neuroscience, vol. 5, no. 2, pp. 146-156, 2004.

[4] Q. Wang, A. Y. Sun, A. Simonyi et al., "Ethanol preconditioning protects against ischemia/reperfusion-induced brain damage: role of NADPH oxidase-derived ROS," Free Radical Biology and Medicine, vol. 43, no. 7, pp. 1048-1060, 2007.

[5] J. M. Mulvey, N. W. C. Dorsch, Y. Mudaliar, and E. W. Lang, "Multimodality monitoring in severe traumatic brain injury: the role of brain tissue oxygenation monitoring," Neurocritical Care, vol. 1, no. 3, pp. 391-402, 2004.

[6] K. Arii, H. Igarashi, T. Arii, and Y. Katayama, "The effect of ozagrel sodium on photochemical thrombosis in rat: therapeutic window and combined therapy with heparin sodium," Life Sciences, vol. 71, no. 25, pp. 2983-2994, 2002.

[7] T. Imamura, S. Kiguchi, K. Kobayashi, K. Ichikawa, Y. Yamazaki, and M. Kojima, "Effect of ozagrel, a selective thromboxane A2-synthetase inhibitor, on cerebral infarction in rats. Comparative study with norphenazone, a free-radical scavenger," Arzneimittel-Forschung, vol. 53, no. 10, pp. 688$694,2003$.

[8] H. Komatsu, Y. Takehana, and S. Hamano, "Beneficial effect of OKY-046, a selective thromboxane A synthetase inhibitor, on experimental cerebral vasospasm," Japanese Journal of Pharmacology, vol. 41, no. 3, pp. 381-391, 1986. 
[9] S. Suzuki, K. Sano, H. Handa et al., "Clinical study of OKY046, a thromboxane synthetase inhibitor, in prevention of cerebral vasospasms and delayed cerebral ischaemic symptoms after subarachnoid haemorrhage due to aneurysmal rupture: a randomized double-blind study," Neurological Research, vol. 11, no. 2, pp. 79-88, 1989.

[10] T. Yoshikawa, Y. Akiyoshi, T. Susumu et al., "Ginsenoside $\mathrm{Rb}$ reduces neurodegeneration in the peri-infarct area of a thromboembolic stroke model in non-human primates," Journal of Pharmacological Sciences, vol. 107, no. 1, pp. 32-40, 2008.

[11] Y. Bu, Z. H. Jin, S. Y. Park et al., "Siberian ginseng reduces infarct volume in transient focal cerebral ischaemia in Sprague-Dawley rats," Phytotherapy Research, vol. 19, no. 2, pp. 167-169, 2005.

[12] H. Y. Son, H. S. Han, H. W. Jung, and Y. K. Park, "Panax notoginseng attenuates the infarct volume in rat ischemic brain and the inflammatory response of microglia," Journal of Pharmacological Sciences, vol. 109, no. 3, pp. 368-379, 2009.

[13] D. W. Kaufman, J. P. Kelly, L. Rosenberg, T. E. Anderson, and A. A. Mitchell, "Recent patterns of medication use in the ambulatory adult population of the United States: the Slone survey," Journal of the American Medical Association, vol. 287, no. 3, pp. 337-344, 2002.

[14] Y. O. Kim, H. J. Kim, G. S. Kim et al., "Panax ginseng protects against global ischemia injury in rat hippocampus," Journal of Medicinal Food, vol. 12, no. 1, pp. 71-76, 2009.

[15] E. Z. Longa, P. R. Weinstein, S. Carlson, and R. Cummins, "Reversible middle cerebral artery occlusion without craniectomy in rats," Stroke, vol. 20, no. 1, pp. 84-91, 1989.

[16] K. Radad, G. Gille, L. Liu, and W. D. Rausch, "Use of ginseng in medicine with emphasis on neurodegenerative disorders," Journal of Pharmacological Sciences, vol. 100, no. 3, pp. 175186, 2006.

[17] S. Y. Han, H. X. Li, C. C. Bai, L. Wang, and P. F. Tu, "Component analysis and free radical-scavenging potential of Panax notoginseng and Carthamus tinctorius extracts," Chemistry and Biodiversity, vol. 7, no. 2, pp. 383-391, 2010.

[18] J. Tian, F. Fu, M. Geng et al., "Neuroprotective effect of 20(S)ginsenoside Rg on cerebral ischemia in rats," Neuroscience Letters, vol. 374, no. 2, pp. 92-97, 2005.

[19] R. Ye, N. Li, J. Han et al., "Neuroprotective effects of ginsenoside Rd against oxygen-glucose deprivation in cultured hippocampal neurons," Neuroscience Research, vol. 64, no. 3, pp. 306-310, 2009.

[20] J. M. Collombet, C. Masqueliez, E. Four et al., "Early reduction of NeuN antigenicity induced by soman poisoning in mice can be used to predict delayed neuronal degeneration in the hippocampus," Neuroscience Letters, vol. 398, no. 3, pp. 337342, 2006.

[21] D. J. Cole, P. M. Patel, R. M. Schell, J. C. Drummond, and T. N. Osborne, "Brain eicosanoid levels during temporary focal cerebral ischemia in rats: a microdialysis study," Journal of Neurosurgical Anesthesiology, vol. 5, no. 1, pp. 41-47, 1993.

[22] A. J. Grau, A. Ruf, A. Vogt et al., "Increased fraction of circulating activated platelets in acute and previous cerebrovascular ischemia," Thrombosis and Haemostasis, vol. 80, no. 2, pp. 298 301, 1998.

[23] F. Van Kooten, G. Ciabattoni, C. Patrono, P. I. M. Schmitz, J. Van Gijn, and P. J. Koudstaal, "Evidence for episodic platelet activation in acute ischemic stroke," Stroke, vol. 25, no. 2, pp. 278-281, 1994.
[24] N. Heye and J. Cervós-Navarro, "Microthromboemboli in acute infarcts: analysis of 40 autopsy cases," Stroke, vol. 27, no. 3, pp. 431-434, 1996. 

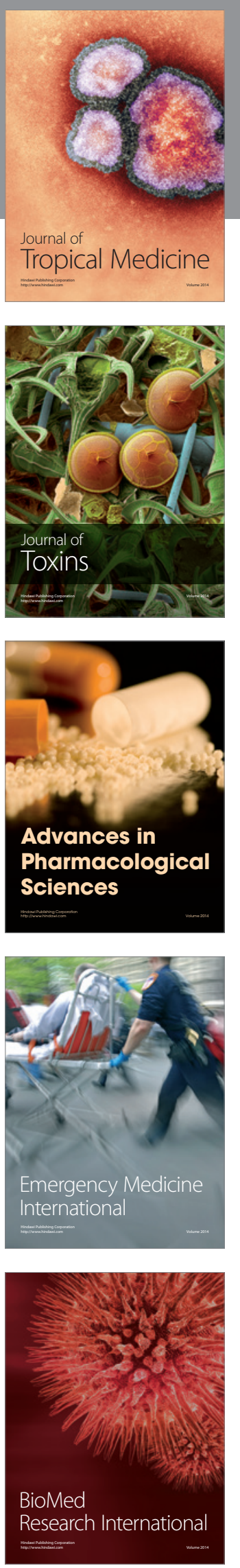
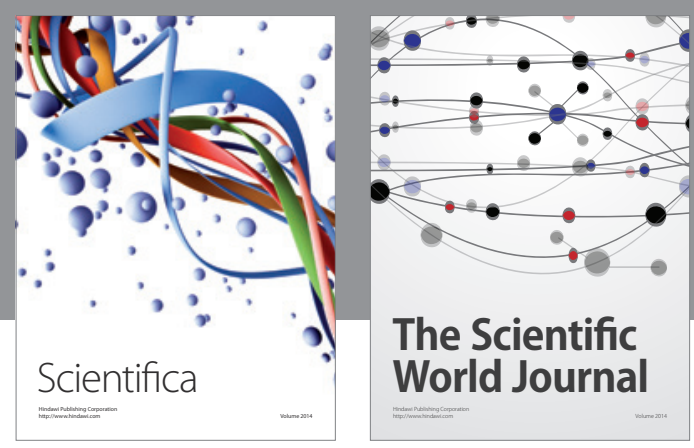

The Scientific World Journal
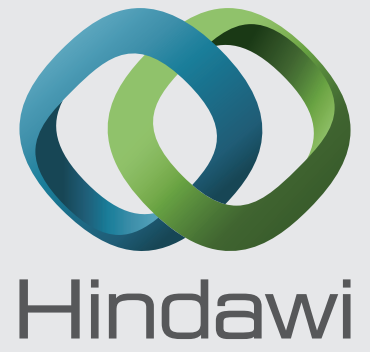

Submit your manuscripts at

http://www.hindawi.com
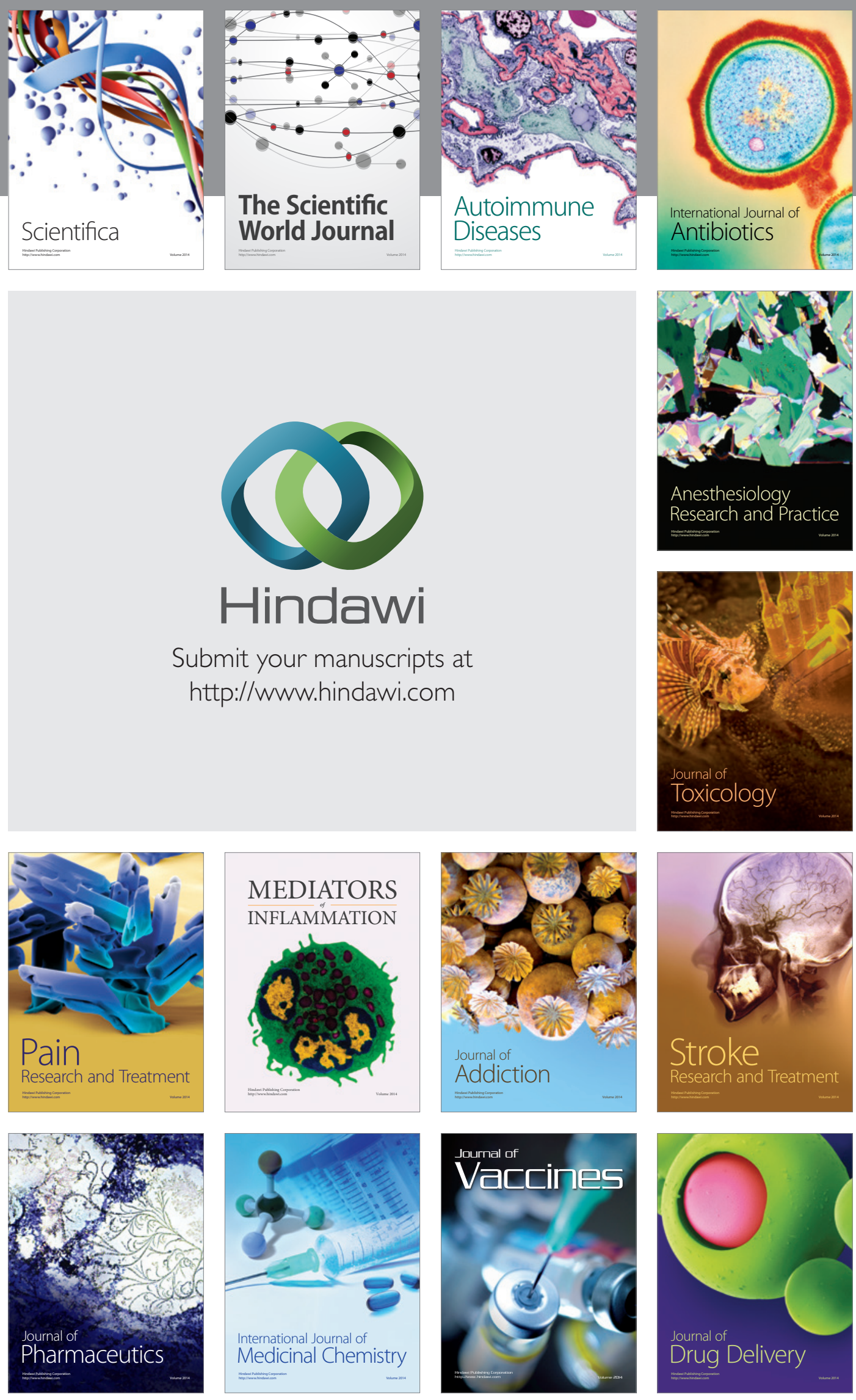